\title{
Experience with a perfusion-only screening protocol for evaluation of pulmonary embolism during the COVID-19 pandemic surge.
}

\author{
Arun Kumar, MD $\quad$ 0000-0002-9127-8459 \\ Renée M. Moadel, MD, MS $\quad$ 0000-0002-0499-8715 \\ Linda B. Haramati MD, MS*+ 0000-0003-0365-7422 \\ Kenny Ye, PhD 0000-0002-1466-855X \\ Leonard M. Freeman, MD $\quad$ 0000-0002-6125-9409 \\ Lionel S. Zuckier, MD, MBA $\quad$ 0000-0002-7212-0437
}

Divisions of Nuclear Medicine ${ }^{\#}$ and Cardiothoracic Imaging*, Departments of Radiology and Medicine ${ }^{+}$, Montefiore Medical Center and Albert Einstein College of Medicine, Bronx, NY 10467 and Division of Biostatistics, Department of Epidemiology \& Population Health , Albert Einstein College of Medicine, Bronx, NY 10461

Corresponding author:

Lionel S. Zuckier, Division of Nuclear Medicine, 1695A Eastchester Road, Bronx, NY 10461

Phone: 718-405-8468 Email: 1 zuckier@montefiore.org

First author (resident in training):

Arun Kumar, Division of Nuclear Medicine, 1695A Eastchester Road, Bronx, NY 10461

Phone: 718-405-8468 Email: arkumar@montefiore.org

Word count: 4495

Financial support: none

No reprints available

Immediate Open Access: Creative Commons Attribution 4.0 International License (CC BY) allows users to share and adapt with attribution, excluding materials credited to previous publications.

License: https://creativecommons.org/licenses/by/4.0/. Details: https://jnm.snmjournals.org/page/permissions.

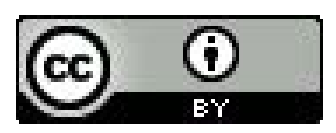




\begin{abstract}
The purpose of this study is to evaluate a pulmonary embolism (PE) perfusion-only screening (POS) protocol, introduced during the pandemic surge. Subjects without dense parenchymal lung opacities were studied; those with $<1$ segmental perfusion defect were considered without PE while patients exhibiting $\geq 1$ defects were indeterminate, mandating additional examinations to determine final diagnosis.
\end{abstract}

\title{
Methods
}

We analyzed demographic information, clinical data, imaging findings and follow-up from electronic records in coronavirus disease, 2019 (COVID-19) patients who underwent lung scintigraphy during the 60-day study period.

\section{Results}

53 studies were performed in 17 COVID-19 positive and 36 COVID-19 negative patients. The POS protocol efficiently excluded PE in $79 \%$ of cases; the remaining $21 \%$, indeterminate for PE, were generally referred for alternative testing or directly anticoagulated. In patients with negative POS studies there was a very low mortality prior to hospital discharge (1/42), and normal follow up studies when performed (6/6).

\section{Conclusion}

The POS protocol, implemented during the COVID-19 surge, efficiently and safely excluding PE in $79 \%$ of patients.

Key words: ventilation; pulmonary embolism; COVID-19; lung scintigraphy

Disclosures: None. No potential conflicts of interest relevant to this article exist. 


\section{INTRODUCTION}

The purpose of this study is to evaluate a screening protocol for pulmonary embolism (PE) which we introduced during the initial coronavirus disease, 2019 (COVID-19) surge (1), which was modeled after an algorithm employed at our institution for evaluation of PE in pregnant women (2). No ventilation scintigraphy is performed. (1)Absence of PE is based on detecting < 1 segmental planar perfusion defects; studies demonstrating $\geq 1$ segmental defects are considered indeterminate for PE mandating additional examinations to determine final diagnosis. Patients with known dense parenchymal lung opacities, in whom corresponding perfusion defects are anticipated, are directly referred to alternate studies, such as CTPA. We hope our(3) experience will help inform the global discussion of best practices during periods of elevated risk from infectious respiratory pathogens.

\section{MATERIALS AND METHODS}

IRB approval was obtained with waiver of requirement for consent. We retrospectively retrieved demographic information, clinical data, imaging findings and patient follow-up from electronic records in patients studied between March 21 and May 19, 2020, coincident with the initial surge of COVID-19 in our high-prevalence region. Continuous variables were summarized as mean \pm standard deviation (SD) while highly skewed variables were described by median and interquartile ranges. Comparisons between the means of continuous variables were performed using Welch's t-test unless otherwise indicated and comparison of proportions was performed using Fisher exact test. P-values $<0.05$ were considered statistically significant.

\section{RESULTS}

\section{Demographic findings}

53 patients underwent lung scintigraphy during the 60-day period. Infection with SARS-CoV-2 virus was evaluated by PCR in 46 patients, 13 positive and 33 negative, while 7 patients were categorized by clinical judgement, 4 infected and 3 not. Demographic and laboratory values are listed in Table 1. 


\section{Prior radiographic findings}

Chest radiographs or CT examinations were obtained in all patients prior to the perfusion study within 0-5 days (mean $0.8 \mathrm{~d}$ ). No dense parenchymal opacities were present however 14 radiographic findings were present in 13 subjects consisting of ill-defined and non-segmental opacities (7 patients), mild congestive heart failure ( 4 patients), and linear atelectatic changes ( 3 patients).

In the 17 COVID-19 positive (COV+) patients, 2 negative Doppler ultrasound studies of the legs were performed preceding scintigraphy. In the 36 COVID-19 negative (COV-) patients, there were 6 negative and 2 positive Doppler studies and 2 nondiagnostic CTPA examinations prior to scintigraphy.

\section{Scintigraphic studies}

Planar perfusion scintigraphy was performed according to SNMMI guidelines (3). Following injection of $148 \mathrm{MBq}$ of ${ }^{99 \mathrm{~m}} \mathrm{Tc}-\mathrm{MAA}$, planar images in 8 projections were acquired, 180s per view. Significant defects were wedge shaped and pleural based with sizing conforming to SNMMI guidelines

(3). Exceptions to standard protocol occurred in 8 patients where reduced dosages of radiopharmaceutical were administered, 2 patients where low dose ventilation was performed prior to scintigraphy, and 4 patients where SPECT-CT was additionally performed. Alterations in imaging confirmed but did not alter diagnoses based on planar perfusion imaging.

Frequency of segmental perfusion defects in the $\mathrm{COV}+$ and $\mathrm{COV}-$ groups on planar perfusion scintigraphy was $18 \%$ and $22 \%$ respectively (figs. $1 \mathrm{a}$ and b); in 6 instances single segmental defects were identified, 4 patients multiple segmental defects were identified, and 1 case there was a relative unilateral decrease in perfusion (fig. 2).

\section{Clinical follow-up in patients with normal perfusion studies}

Of 14 patients with normal perfusion in the COV+ group, 12 were not studied further while 2 underwent Doppler ultrasonography of the legs, both negative (fig. 1a). Three patients were placed on anticoagulation, one of whom had atrial fibrillation. One of the 11 non-anticoagulated patients died of respiratory failure during hemodialysis 2 days following scintigraphy; the remaining $13 \mathrm{COV}+$ patients with normal perfusion were successfully discharged home. 
Of 28 patients with normal perfusion studied in the COV-group, 24 were not studied further while 2 underwent CTPA, 1 Doppler US of the legs, and 1 CTPA followed by Doppler US, all negative (fig. 1b). Three patients with normal perfusion in this group were anticoagulated, 1 with prior positive leg Doppler ultrasound, 1 with history of prior PE 4 years earlier, and 1 in atrial fibrillation. All 28 patients with normal perfusion in the COV- group were discharged home.

\section{Clinical follow-up in patients with indeterminate perfusion studies}

None of the $3 \mathrm{COV}+$ patients with perfusion defects received any follow-up examinations and all were directly anticoagulated (fig. 1a), one of whom died of respiratory failure a day post scintigraphy. Six out of $8 \mathrm{COV}$ - patients with indeterminate findings were referred for further diagnostic imaging demonstrating 1 positive catheter angiogram, 1 negative Doppler US of the legs followed by positive CTPA, and 4 negative CTPA examinations (fig. 1b). Another COV-patient with indeterminate findings had a prior positive Doppler study and was directly anticoagulated without additional imaging or adverse outcome. The final patient had a perfusion defect described as atypical and was discharged home without therapy. The patient who underwent catheter angiography had contraindication to Heparin; thrombolysis was performed with placement of an IVC filter however she died of multisystem failure 11 days thereafter. The patient with positive CTPA was anticoagulated while 4 patients with negative CTPAs were not. Of all 11 patients with indeterminate perfusion studies, 9 patients were therefore ultimately discharged home (2 COV+ and $7 \mathrm{COV}-$ ).

\section{DISCUSSION}

A variety of approaches to performing lung scintigraphy were considered during the early COVID-19 period (4), designed to balance tension between potential spread of infection when ventilation scintigraphy is performed and sub-optimal specificity of scintigraphy when ventilation is omitted.

(6)These considerations informed our approach, which utilized perfusion scintigraphy in a screening role(2), relying on the established sensitivity of perfusion scintigraphy to identify disease and not creating new criteria of interpretation. (6-8) 
The most salient observation regarding the POS protocol is that $\sim 80 \%$ of patients had $<1$ segmental defect and required no further testing. (9)By restricting the patients that we studied to those with a relatively clear chest radiograph, we succeeded in obtaining a subgroup of subjects with low prevalence of defects, thereby excluding PE in an efficient manner and validating anticipated benefit of the protocol. A subgroup of 13 patients were imaged in spite of presence of mild parenchymal abnormalities which did not interfere with performance of the examination.

The POS protocol was accurate and safe. Of 6 patients who underwent additional diagnostic examinations following a negative study, no emboli or thrombi were confirmed. Amongst all 42 patients with a negative POS study there only was 1 fatality, a COV+ patient who died of respiratory failure. (7)Of the 11 subjects with indeterminate perfusion scintigraphy, 6 underwent further testing of which 2 patients had PE substantiated on follow-up, demonstrating use of POS as a screening examination. One $\mathrm{COV}$ - patient with proven embolism and contraindication to anticoagulation succumbed to multisystem failure while a second $\mathrm{COV}+$ patient who was placed on anticoagulation died of respiratory failure 1 day following scintigraphy.

Limitations of our retrospective study include inability to determine outcome by optimal goldstandard of 60-day follow-up because most patients were not enrolled within our healthcare system postdischarge. As well, we cannot easily reconstruct how many patients were referred away from scintigraphy due to radiographic abnormalities or other considerations and which alternative examinations they underwent. A final limitation relates to the generalizability of our findings. Prevalence of segmental defects in patients referred for testing is highly dependent on specific referral patterns and regional incidence of disease. Nonetheless, the relatively robust results we obtained suggests that this protocol could be cautiously extrapolated to similar environments with ongoing monitoring of efficacy.

While we imaged by planar scintigraphy, a similar screening protocol can be constructed based on SPECT imaging, employed in many regions (5). It is also possible that interim specificity can be improved by factoring in pretest probability (6) or by performing SPECT-CT, where anatomic CT information can be used as a partial surrogate for ventilation (6-8). 
An intriguing consideration deriving from our experience is whether performing a perfusion study first as a screening test should continue in non-infected patients with relatively clear chest radiographs in whom PE is being excluded (9). Only if segmental defects are noted on the perfusion study would a completion ventilation study or complimentary imaging be performed.

\section{CONCLUSION}

We have reviewed our experience during 60 days of the initial COVID-19 surge employing a perfusiononly lung scan protocol implemented in 53 patients with minimal abnormalities on baseline chest radiograph. This screening protocol efficiently and safely excluded PE in $79 \%$ of patients studied.

\section{FINANCIAL DISCLOSURE}

None

\section{ACKNOWLEDGEMENTS}

We gratefully acknowledge the selfless contribution of our technologists and front-line staff who faithfully provide compassionate and expert care. 


\section{KEY POINTS}

Question: Is the perfusion-only screening algorithm, introduced during the COVID-19 surge to assess for pulmonary embolus, an efficient and safe method of evaluation?

Pertinent findings: We retrospectively analyzed chart data in 57 patients studied over the initial 60 days of the COVID-19 surge. Perfusion-only imaging was able to efficiently exclude PE in $79 \%$ of patients and was accurate and safe as determined by follow-up imaging and hospital discharge.

Implications for patient care: Perfusion-only screening protocols can be safely implemented in situations where ventilation scintigraphy cannot be performed and serve to efficiently exclude PE in a large majority of patients. 


\section{REFERENCES}

1. Zuckier LS, Moadel RM, Haramati LB, Freeman LM. Diagnostic Evaluation of Pulmonary Embolism During the COVID-19 Pandemic. J Nucl Med. 2020;61:630-631.

2. Sheen JJ, Haramati LB, Natenzon A, et al. Performance of Low-Dose Perfusion Scintigraphy and CT Pulmonary Angiography for Pulmonary Embolism in Pregnancy. Chest. 2018;153:152-160.

3. Parker JA, Coleman RE, Grady E, et al. SNM practice guideline for lung scintigraphy 4.0. J Nucl Med Technol. 2012;40:57-65.

4. Zuckier LS. To everything there is a season: taxonomy of approaches to the performance of lung scintigraphy in the era of COVID-19. Eur J Nucl Med Mol Imaging. 2020:1-4.

5. Le Roux PY, Pelletier-Galarneau M, De Laroche R, et al. Pulmonary Scintigraphy for the Diagnosis of Acute Pulmonary Embolism: A Survey of Current Practices in Australia, Canada, and France. J NuCl Med. 2015;56:1212-1217.

6. Miniati M, Monti S, Bauleo C, et al. A diagnostic strategy for pulmonary embolism based on standardised pretest probability and perfusion lung scanning: a management study. Eur J Nucl Med Mol Imaging. 2003;30:1450-1456.

7. Das JP, Yeh R, Schöder H. Clinical utility of perfusion (Q)-single-photon emission computed tomography (SPECT)/CT for diagnosing pulmonary embolus (PE) in COVID-19 patients with a moderate to high pre-test probability of PE. Eur J Nucl Med Mol Imaging. 2020:1-6.

8. Lu Y, Macapinlac HA. Perfusion SPECT/CT to diagnose pulmonary embolism during COVID-19 pandemic. Eur J Nucl Med Mol Imaging. 2020;47:2064-2065.

9. Boone SL, Zuckier LS. VQ Scans After the COVID-19 Era Point-Ventilation Studies Are Dispensable. AJR Am J Roentgenol. 2022, in press. 
17 COVID-positive patients

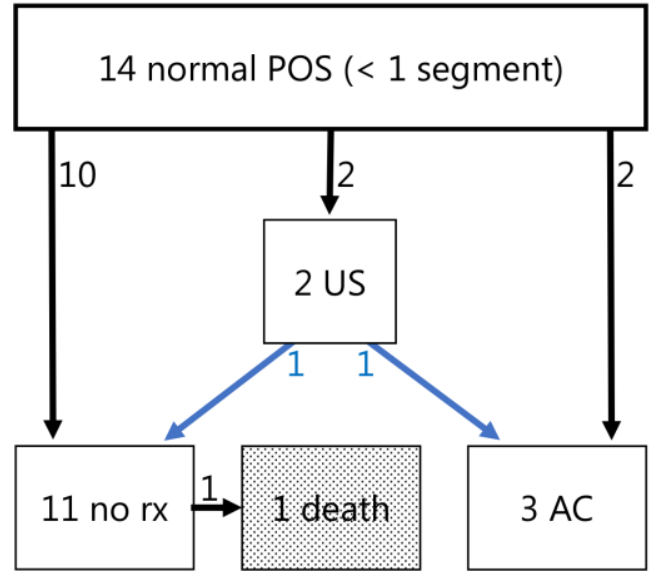

3 indeterminate POS ( $\geq 1$ segment)

Perfusion

scintigraphy

Subsequent

procedures

36 COVID-negative patients
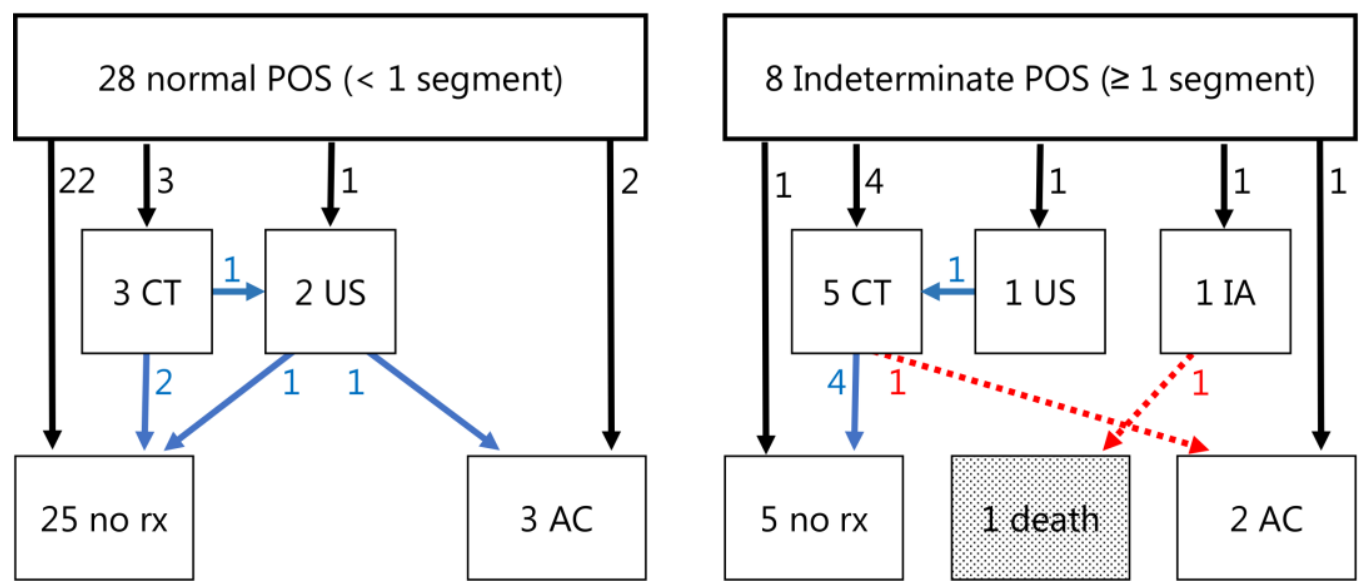

Perfusion scintigraphy

Subsequent procedures

\section{Outcomes}

Figure 1. Diagnostic flowchart in $17 \mathrm{COV}+($ Fig. 1A) and $36 \mathrm{COV}-($ Fig. 1B) patients. Arrows refer to the flow of patients while adjacent numbers indicate the number of patients involved. Blue solid arrows indicate a negative test result; red dotted arrows signify a positive test result. Abbreviations: POS - Perfusion-only screening; CT - CTPA; US - leg Doppler ultrasound; IA - Interventional Angiography; rx - therapy; AC - anticoagulation. 


\begin{tabular}{|l|l|l|l|l|}
\hline Chest X-ray & Anterior & Posterior & Left Post Oblique & Right Post Oblique \\
\hline A & & & & \\
\hline B & & & & \\
\hline & & & & \\
\hline
\end{tabular}

Figure 2. Key images in 4 representative patients. All chest radiographs demonstrate absence of significant opacities. A. 44-year-old woman, COV+ by PCR. No defects were noted on perfusion scintigraphy. The patient was not anticoagulated and was discharged without complication. B. 35-year-old woman, COV- by PCR. Well-defined segmental perfusion defect in the superior lingula, indeterminate for PE. CTPA demonstrated normal pulmonary arterial perfusion; patient was discharged home without anticoagulation. C. 43-year-old man, COV+ by PCR, with elevated D-dimer $(19.7 \mu \mathrm{g} / \mathrm{mL})$. Multiple bilateral segmental defects, especially involving the right lung, indeterminate for PE. Patient subsequently was discharged on anticoagulation. D. 59-year-old woman, COV- by PCR. There is global decrease in perfusion of the right lung, indeterminate for PE. CTPA demonstrated normal pulmonary arterial perfusion; patient was discharged home without anticoagulation. 


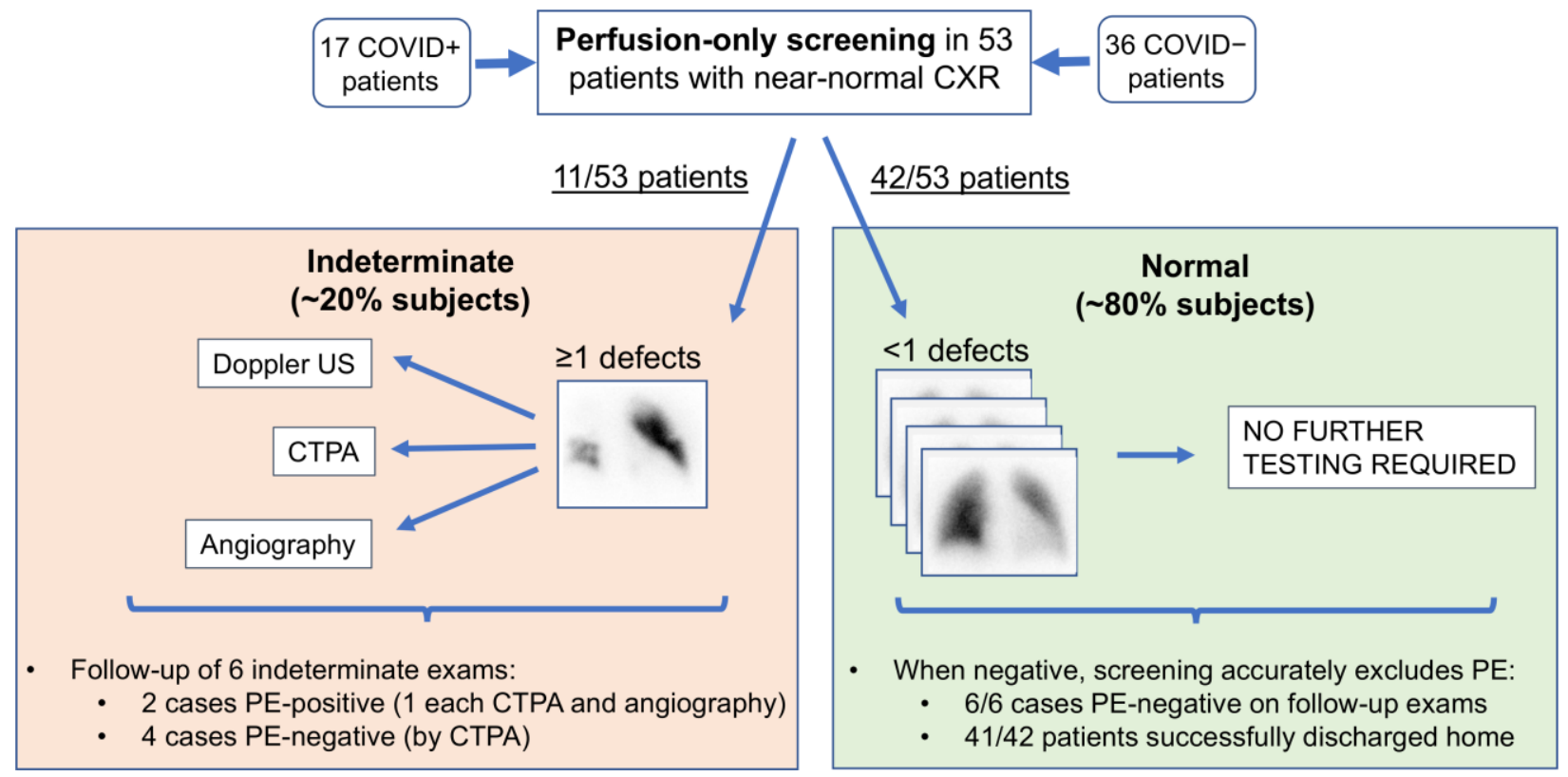

\section{Graphical Abstract}


Table 1. Demographic and clinical findings

\begin{tabular}{|c|c|c|c|c|}
\hline Parameter & All Patients & COVID-19 Positive & COVID-19 Negative & $\begin{array}{c}\mathbf{P} \\
\text { value } ~\end{array}$ \\
\hline - COVID-19 status (by PCR) & 46 & 13 & 33 & - \\
\hline Age in years (mean \pm 1 S.D.) & $49.0 \pm 16.2$ & $47.5 \pm 17.2$ & $49.8 \pm 15.9$ & 0.68 \\
\hline Females $(\%)$ & $39(74 \%)$ & $10(59)$ & $29(81)$ & 0.11 \\
\hline Patients with serum creatinine $\geq 1.5 \mathrm{mg} / \mathrm{dL}(\%)$ & $19(36 \%)$ & $6(35 \%)$ & $13(36 \%)$ & 1 \\
\hline Parenchymal findings on prior chest radiography (\%) & $13(25 \%)$ & $7(41 \%)$ & $6(17 \%)$ & 0.08 \\
\hline Patients with prior negative Doppler US & 8 & 2 & $6^{*}$ & - \\
\hline Patients with prior positive Doppler US & 2 & 0 & 2 & - \\
\hline Patients with prior nondiagnostic CTPA & 2 & - & $2 *$ & - \\
\hline Patients with $\geq 1$ perfusion defects $(\%)$ & $11(21 \%)$ & $3(18 \%)$ & $8(22 \%)$ & 1 \\
\hline
\end{tabular}

\section{LEGEND}

$\sim \mathrm{P}$ value of differences between the COVID-19 positive and the COVID-19 negative subgroups

\# In $\mu \mathrm{g} / \mathrm{mL}$, normal value $\leq 0.50 \mu \mathrm{g} / \mathrm{mL}$. P value based on Wilcoxon rank-sum test.

* One patient had both negative Doppler exam and nondiagnostic CTPA. 